\title{
Intelligent analysis in construction management
}

\author{
Fjoder Klashanov ${ }^{1, *}$ \\ ${ }^{1}$ Moscow State University of Civil Engineering, Yaroslavskoye shosse, 26, 129337, Moscow, Russia
}

\begin{abstract}
The article is devoted to analysis of data mining techniques in a model of construction management. In the construction industry, as a rule, in most cases is incomplete and/or unclear information, so the classical methods of model-building control don't work. In this case, it is advisable to apply some well developed methods of fuzzy sets and fuzzy logic. The original construction is described by the words of the spoken language, where the sentences are predicates of the first kind. Identifies the parameters that influence the management process in the form of a lexical variable, which is analyzed by methods of the theory of many-valued logic. The results of the analysis are used in building the model administration of construction.
\end{abstract}

\section{Introduction}

Construction production, being the most important branch of successful development of the country, imposes the corresponding requirements to its organization, the information description and processing of data of information fields. Therefore, in the modern world to the construction industry more and more requirements. Construction projects must meet all the requirements that meet the comfort, safety, environmental and at the same time efficiency. The task of building information flow management is multifunctional, and appropriate methods are needed to solve it. Given the fact that the information about the construction is fuzzy and/or incomplete, the well-proven cybernetic methods for clearly defined tasks are not suitable. First-order logic for knowledge representation in areas such as construction is not applicable because of the complexity and labor input of forming a complete set of antecedents or consequents. Therefore, preliminary analysis of the structure and stages of the entire life cycle of the real estate object is carried out, and then for each stage (stage) the method of analysis of the relevant information is determined using the developed theory of artificial intelligence systems. The life cycle of a real estate object as a construction object is a sequence of processes of existence of the real estate object from conception to liquidation (disposal). Here it is necessary to solve economic problems in the development of the investment cycle and the material and energy problems and many other heterogeneous problems.

Management in the construction industry is being produced, the higher the requirements set out in the relevant normative documents. It is primarily the efficiency, safety, comfort and environmental friendliness, which should be ensured at all stages of the life cycle of construction object. But the information has the developer, as the rules unclear and/or

\footnotetext{
*Corresponding author: klashanov@mgsu.ru
} 
incomplete. Therefore, classical methods based on cybernetic approach in this case is not applicable. In this case, it is necessary to apply methods of artificial intelligence systems and to use methods based on two-valued logic, multivalued and fuzzy sets [1]. In practice, rarely encountered a situation when they are described fully and clearly, therefore, the conclusions based on them cannot be considered absolutely true. In the case of definite descriptions apply classic techniques - binary logic, and otherwise fuzzy logic. In the case of classical logic the conditions and conclusions can be either absolutely true or absolutely false; in fuzzy logic may be a partial truth, with the value belonging to the interval $(0,1)$.

Methods of fuzzy logic are used in various industries, for example, for quantitative risk assessment of gas distribution networks, methods for the determination of design loads for both the main and distribution pipelines are based on fuzzy input data [2]. All the pipelines included in the distribution network is divided into main and distribution. Gas pipelines mainly perform the function of transporting gas within the city, and gas distribution pipelines are used to supply gas directly to consumers, although this division is conditional. The man himself determines the necessary number of terms and each of them puts in conformity to some value described by physical quantities. For this value the degree of belonging to the physical quantity term is equal to unity and all other values depending on the chosen membership functions.

In States that "the property obliges scientists to build their research in the light of chaos theory and Heisenberg's uncertainty principle, which state the impossibility of accurate forecasting and measurement of components in complex systems In the analysis of the sintering zone of rotary cement kiln also applies methods of multiple-valued logic as industrial oven - thermal apparatus and is described primarily thermodynamic and aerodynamic parameters, which are fully and unambiguously determined. A similar problem arises in studies of the sensory control of motors, and also in the calculation of the ventilation system in the self-constructions. The design of the instrumentation to detect the position of the rotor of the motor uses Chistiakova (or trapezoidal) commutation algorithm based on linguistic variable. Problems that need to be addressed in the development of future corporate memory for knowledge management for the problems of the industry[8,9], also based on fuzzy logic. Knowledge management is considered as a collection of processes that govern the creation, dissemination, processing and use of information within the enterprise [10]. What has traditionally had to deal with automated system control, which lacked internal consistency, give the logical-linguistic models [11]. Knowledge is the result of processing and development of any information, the use of which allows to obtain a particular result, a certain predetermined way. The use of knowledge allows you to turn in the work required tangible and intangible resources into the desired product.

\section{Materials and Methods}

In task construction management, has to deal with the set of feasible solutions among which the optimum is sought, which must be found. There are many methods, the most frequent and well-developed cybernetic or classic method, which is applied if a building is not an object of high complexity and operates in a completely deterministic environment, which is uniquely described by a number of parameters. For these settings a functional dependency, which may be different, but deterministic [12]. Holds the formalization of the control object, namely, that highlighted the parameters that describe the object and are mounted between functional relationships. The real object is replaced by its mathematical model representing the control object. Based on this mathematical model, the composed algorithm, which are then implemented on a computer operating on two-valued logic, using only such concepts as TRUTH (YES) and FALSE (NO), or how it is interpreted in computing the one and zero [13]. 
In real life the concept of only YES or NO are extremely rare. Real formal problem to resolve with the required accuracy on the computer, it is necessary to describe the parameters of the degree of truth, which is not subject to binary logic except zero and one there are concepts that have a degree of certainty belonging to the segment from zero to one. To solve the control problem by classical methods with a given accuracy is impossible because of the incorrect description of the source data, even if the mathematical model will not be difficult. Double-digit logic for problems described by ordinary spoken language, makes it impossible to formulate the problem for a computer[13,14]. For example, our spoken language allows on the basis of classical logic to enter into the computer are concepts that are expressed with an adjective. If you will be told that for the production of these earthworks are looking for a POWERFUL excavator to formalize this requirement for your computer based on binary logic, impossible. The word POWERFUL is not clearly defined. This is because the logic of Aristotle is based on the postulate of the Tertium non datur ("a Third is not given" - lat.) that is if the statement is true, then it is not false.

In this case, something in between truth and falsehood, i.e. intermediate values between zero and one excluded. But it does not correspond to the real situation. If you need to lift a heavy load, will require a heavy lift crane. But with Aristotelian logic to describe the GREAT impossible. In the development of the management system in the construction of the original source of information are the words of a natural language, giving a qualitative assessment belonging to some universal set E, determined by expert way. For example, variables can be: load, speed, distance, budget values, which follow from experience.

They can be described by such fuzzy concepts as LARGE, MEDIUM, SMALL, APPROXIMATELY ABOUT. So, the value of the variable load can be - VERY easy, EASY, difficult, VERY difficult, and distance can be described by the concepts of FAR, NEAR, etc. a Verbal description of the control object is performed by means of sentences of predicates which are linguistic variables. The term "linguistic variable" to describe any value that you want to have more values than just YES and NO. To do this, define the required number of terms and each of them corresponds to a certain value, for example for shipping: VERY EASY, EASY, NORMAL, hard, VERY HARD. Now for each term the degree of belonging, which will be equal to one for the term, and all other values are calculated according to the selected membership functions. Currently for the description of linguistic variables, terms and fuzzy rules uses a special language: Fuzzy Control Language (FCL) described in the standard IEC 1131.

In the case of finding managerial solutions in the construction industry requires the use of unambiguous logic, and multivalued with the use of fuzzy sets. Multivalued logic and fuzzy set gives you the opportunity to move from linguistic variables to a numeric equivalent analogues, which give the ability to use digital computers. Classical logic when representing knowledge is not clearly formulated objectives are not applicable due to the fact that the formation of the full set of antecedents or consequential is too complex and it is impossible to consider the full uncertainty of all kinds of production situations.

These concepts define the fuzzy linguistic variable taking any value from the set E, called the baths. Thus, any variable in fuzzy logic is uniquely identified not by numbers, and terms $\mathrm{T}$ that are subsets of $T \subset E$. All of these requirements, first of all, is relatively easy to formulate verbally in the form of declarative sentences with a definite answer first-order predicate that takes values YES or NO (1 or 0). But not all the factors based on which you need to take a managerial decision, you can describe on the basis of two-valued logic [15]. For example, the supply of construction materials from a specific vendor within a specified period and with certain financial costs clearly answer YES or NO not always possible. In this case, the management decision it is necessary to use fuzzy logic. Fuzzy logic and fuzzy sets, as shown in mathematics are a generalization of the classical twovalued logic and crisp sets. 
The notion of a set in fuzzy logic augmented with the introduction of membership function. Each element of the set is assigned a certain value lying in the interval [0...1]. This value takes the membership function defines the degree of truth (confidence) of a specific value to a given term. To clear set the value of this function takes only two values: a unit - element belongs to set and the zero element does not belong to the set. Developed rules for operations on fuzzy sets; they are similar to the operations on crisp sets, but their specific features. The main advantage of fuzzy logic is that it relies on the use of linguistic revolutions natural language. It has found application in the management of the appliances and the control of complex industrial objects and processes.

Fuzzy logic uses concepts such as "fuzzy rules, fuzzy inference" and the term "fuzzy control". Formalization of the linguistic terms for input variables, allows writing the fuzzy rules in the form "if $X$ then $Z$ " where $X$, as a source of primary information. The second part of the rule $Z$ is the result, which corresponds to the desired control action. The first part $\mathrm{X}$ is a linguistic term that contains the primary information of the expert about the studied object.

Fuzzy sets are described by multivalued logic that allows solving tasks on the compute machines. To use this method the notion of linguistic variable, which can be applicable to any physical quantity, the characteristics of which require more values than just YES and NO. This is implemented in the following way. Let $U$ be the universal set, $X$ element of $R$, and some property of this set. A clear subset of A universal set whose elements satisfy property $\mathrm{R}$, is defined as an ordered set of pairs $A=\left\{\mu_{A}(x) / x\right\}$ where $\mu_{A}(x)$ the characteristic function, taking value 1 if the element satisfies the property $\mathrm{R}$, and 0 otherwise. For the fuzzy subset of elements it does not answer unequivocally, "YES-NO" regarding the properties of R. a Fuzzy subset of a universal set is a set of ordered pairs of $\mathrm{x}$ and where the characteristic membership function taking values in the set $M=[0,1]$. The membership function indicates the degree of belonging of element $\mathrm{x}$ to A. many of Its extreme values 0 not belongs to the set $\mathrm{A}, 1$ - belongs to the set $\mathrm{A}$. the set $\mathrm{M}=[0,1]$ indicates the degree of certainty of $\mathrm{x}$ belonging to the set $\mathrm{A}$ and changes as well as changes the value of probability is a common and clear set. The set of elements $\mathrm{X}$ for which $\mu_{A}(x)>0$ is a carrier of a fuzzy set supp A: supp $\mathrm{A}=\left\{x \in X ; \mu_{A}(x)>0\right\}$.

Thus, the exact membership of each value to one of the terms of a linguistic variable is defined by a membership function $\mu_{A}(x)$. The function can be arbitrary, although there is the concept of standard membership functions, which include functions such as Z-function, $S$-function, triangular function and trapezoid function. These functions are used to solve many problems. In each case, can be used and other types of functions, and other methods of fuzzy logic.

So in the expert systems use production rules that allow to take into account the associative specificity of human thinking. The expert approach relies on the introduction of, for example, such linguistic terms as "low", "medium", "elevated", "high".

Rule products are the parcels and the conclusion of the ligaments combined with logical AND, OR. This allows the rule to record in the form used in cybernetic calculation: "IF (the parcel) (ligament) (premise) (premise) THEN (conclusion)". One of the disadvantages of production systems is that their effective operation requires full information about the object. In fuzzy systems, is also based on production rules, but they use linguistic variables, thus avoiding the difficulties associated with the completeness of the description of the object of study, as in the classical production rules. For these purpose mathematical methods of transition from the fuzzy values of variables to well defined values. It can be argued that fuzzy logic generalizes classical two-digit logic of reasoning under uncertainty. It gives you the opportunity to describe imprecise qualitative concepts and making them measurable through the introduction of linguistic variables. 


\section{Results}

The process of fuzzy control is divided into several stages, among which are the following three: fuzzification, development of fuzzy rules and defuzzification. Then the processor that implements this algorithm, processes data from the sensors according to the principle similar to the human concept of allowing a number of intermediate values and not on the binary rules, so the required solution is more correct and adequately reflects the real situation. This algorithm comes with a pack of fuzzy TECH. For example, a model of a tower crane using fuzzy logic can be solved quite easily. Instead of considered algorithm is based on the analysis of systems of algebraic and/or differential equations of the motion described by natural language terms. In this case, fuzzy logic is based on the use of such revolutions in natural language as "heavy", "light", "high", "low", etc. of these terms of atoms using the modifier "not", "very", "extremely", "not very", etc. you can create many other terms.

To practically implement the whole process of fuzzy control can be divided into several stages.

The first step is fuzzification (transition to fuzziness). To introduce a linguistic variable necessary to determine the precise meaning of its terms. For example, a variable LOAD, lifted by the tower crane can take any value from 0 up to 5 tons. Each value of weight from 0 to 5 tons can be assigned to a number from zero to one, which determines the degree of affiliation a given weight (say, 10 meters) to a particular term of a linguistic variable WEIGHT. In our case, weight 4 tons, you can set the degree of belonging to term "heavy", 0.85 , and the term "light" is 0.15 . Specific determination of the values of the membership function, i.e. the degree of belonging is determined by the experts. For most applications it is sufficient 3-7 of terms for each variable. This action allows qualitative fuzzy information to enter into the computer.

Give a verbal description of sequence of actions of holding the fuzzification of formalizing the problem in terms of fuzzy logic.

Step 1. Each term of a linguistic variable put in the corresponding numeric value used to represent this term. The numerical values belong to the interval $[0 ; 1]$.

Step 2. To determine the value of the parameter with the identity " 0 " and " 1 " to this term.

Step 3. After determining the extreme values is necessary to determine intermediate values. They are usually P - or L-functions of number of standard membership functions.

Step 4. For values corresponding to extreme values of the parameter selected S - or Zmembership function

Now carry out the reverse operation, called defazifikatsii.

At this stage, defuzzification is carried out the transition from the fuzzy values of certain physical parameters which can serve as a command to the Executive device.

Second, we perform the development of fuzzy rules. At this stage, want to find production rules that associate the selected linguistic variables. The combination of these rules provides an opportunity to develop a management strategy. A typical rule consists of the antecedent (IF ...) and consequent (THEN ...). The antecedent may contain more than one parcel. In this case, they are combined by logical connections AND or OR.

Fuzzy inference, which is obtained after the computation of fuzzy rules can be divided into two stages - this is a generalization and conclusion. In the first stage of inference determines the degree of belonging of all antecedent rules. For this purpose, in fuzzy logic there are two operators: $\operatorname{MIN}(\ldots) \operatorname{MAX}(\ldots)$. The first calculates the minimum value of degree of membership and the second maximum value. The use of the operator MIN(...) or MAX(...), depends on which ligament is connected parcels in rule. If you use the bundle And apply the operator MIN(...). If the bundle OR apply the operator MAX(...). note, if a 
rule has only one premise, the operators do not need. The next step is conclusion. Similarly, the operators MIN/MAX calculates the value of the consequent. Introductory information are the results of the calculation in the previous step values of belonging antecedents of the rules. The vagueness of the result of the calculation is adjusted by the following methods: method of the center of maximum (CoM) method is highest values (MOM), the method of centroid (CoA).

The theory of fuzzy logic and fuzzy sets can be applied for the creation of the expert modules of the automated systems of diagnostics of a condition of construction equipment, as well as procedures for management of construction production.

Practically this can be realized in a CAD system using fuzzy TECH 3.0, which consists of four phases: system description, Off-line optimization On-line optimization, implementation. A brief description of each stage.

At the stage of system description is the formalization of the problem by means of fuzzy TECH. First of all, it is necessary to describe the used linguistic variables and their membership functions; then describe the control strategy by fuzzy rules that are to be combined into a single database rules or knowledge about the system.

At the stage of Off-line optimization, check the efficiency of the established system by all means of fuzzy TECH. For example, to use a software simulator of the controlled object. For communication of the control system model uses a custom designed communication Protocol fTlink. Based on Windows messaging contact is made with the model.

\section{Discussions}

On stage On-line optimization of developed control system and real control object connected to the physical communication line. At this stage it is possible to observe the behavior of the system in real conditions and if necessary make changes to the control system [14].

At the stage of Implementation need to get the final version of the code, consisting of three main parts: the code of the library functions; the segment of the rule base and membership functions; functions of fuzzy system. The basis of the software code that is generated by fuzzy TECH is a hardware-focused on a specific type of processor core. Included with the package fuzzy TECH MCU-96 software core compatible with several controllers.

Fuzzy control is particularly useful when the information is submitted efficiently, it is not entirely correct, no accuracy required, completely uncertain or when processes are too complex for analysis using conventional quantitative methods developed in the mathematical analysis. It should be noted that fuzzy logic is based on fuzzy control is closer in spirit to human thinking and natural language than traditional logical systems. Fuzzy logic methods give effective tools to take into account uncertainties and inaccuracies is really this problem. Mathematical methods developed in fuzzy logic for fuzzy input information real tasks allow you to build a model adequate to the reality that adequately would be perceived by the computer.

Let us mention some advantages of fuzzy compared to the other methods that also can be found in the publications:

- ability to operate with fuzzy input data: for example, continuously time-varying values (the dynamic problem), the values that cannot be set uniquely (statistical surveys, advertising companies, etc.);

- the ability of fuzzy formalization of criteria of evaluation and comparison, the operating criteria of "majority", "probably", "mostly";

- ability to conduct quality evaluations, as input data, and output results; 
- the possibility of rapid modeling of complex dynamic systems and their comparative analysis with a given degree of accuracy: in terms of principles of behavior of the system described fuzzy methods, first, do not spend a lot of time figuring out exact values of variables and compilation of the descriptive equations, and secondly, it is possible to estimate different variants of the output values.

Consider the advantages of fuzzy logic.

1. Fuzzy logic supports the development of a rapid prototype of a technical device with the further complication of its functionality.

2. Fuzzy logic model more easy to understand than a similar mathematical model based on mathematical analysis.

3. A fuzzy model is more simple implementation in comparison with classical control algorithms for technical systems.

\section{Conclusion}

Make the following conclusions. The scope of fuzzy logic models is extremely broad: it can be applied in the management of technological processes in the construction industry, and in the analysis of financial flows, to restore order in inventory control, logistics and sales of products, faster to calculate wages in a timely manner to take statements.

Analysis of fuzzy decision-making methods allows to formulate the requirements for further developments in this area. This development of theoretical approaches to the description of complex relationships between the criteria, the broader application of intelligent methods based on fuzzy logic and the development of combined decisionmaking methods using fuzzy concepts.

Note that fuzzy logic does not replace conventional control technology, and is complimented by its highly efficient methodology, the implementation of strategies for multivariable control. Thus, the main potential of fuzzy logic lies in the sphere of implementation of functions of Supervisory management.

Despite the fact that fuzzy logic is relatively new, it has already established itself as a relatively simple, reliable and fast theoretical tool to solve problems, not treated with the classic two-digit logic.

\section{References}

1. S. Russell, R. Norvig, Artificial Intelligence (Pearson, 2003)

2. D.A. Yudin, V.Z. Magergut, E.P. Dobrinskiy, World Applied Sciences Journal 24(11), 1460-1466 (2013)

3. P. Suganya, S. Priyadharshini, S. Saranya, National Conf. on Research Advances in Communication, Computation, Electrical Science and Structures (2015)

4. V. Murgul, D. Vuksanovic, N. Vatin, V. Pukhal, Applied Mechanics and materials 680, 524-528 (2014)

5. B.K. Ahirwal, K.K. Pandey, J.S. Bhadoriya, Intern. Journ. of Advanced Research in Electrical, Electronics and Instrumentation Engineering 3, 11 (2014)

6. O. Kuhn, A. Abecker, Corporate Memories for Knowledge Management in Industrial Practice: Prospects and Challenges (1998)

7. T.R. Gruber, Knowledge Acquisition 5(2), 199-220 (1993)

8. B. Tschaitschian, A. Abecker, A. Schmalhofer, Putting Knowledge Into Action: Information Tuning With KARAT, EKAW-97 (1997) 
9. K. Wiig, Knowledge management is no illusion!, Proc. of the First International Conference on Practical Aspects of Knowledge Management (Zurich, Swiss Information Society, 1996)

10. F. Klashanov, To theoretical base of the building to models of management in construction, conferences.dce.ufl.edu/ICCCBE2014 (2014)

11. A.A. Volkov, S.N. Petrova, Information systems and technologies in construction (MGSU, Moscow, 2015)

12. F.K. Klashanov, Jour. Economics and entrepreneurship 1(1), 637-642 (2016)

13. E.A. Statsenko, A.F. Ostrovaia, T.A. Musorina, M.I. Kukolev, M.R. Petritchenko, Magazine of Civil Engineering 68, 86-91 (2016), doi:10.5862/MCE.68.9

14. A.A. Volkov, S. N. Petrova, Information systems and technology in construction, Textbook (MGSU, Moscow, 2015) 